\title{
A Smart Nocturnal Environment Monitoring System for the Hearing Impaired
}

\author{
John Finlay \\ Ulster University \\ Shore Road, N'abbey \\ Co.Antrim, BT370QB \\ Finlay-J11@email.ulster.ac.uk
}

\author{
Stefan Greppmair \\ Ulster University \\ Shore Road, N'abbey \\ Co.Antrim, BT370QB \\ greppmair-s@email.ulster.ac.uk
}

\author{
Michael Jennings \\ Ulster University \\ Shore Road, N'abbey \\ Co.Antrim, BT370QB \\ jennings-m5@ulster.ac.uk
}

\author{
Matthew Kane \\ Ulster University \\ Shore Rd., N'abbey \\ Co.Antrim, BT370QB \\ kane-m16@email.ulster.ac.uk
}

\author{
Johannes Leopold \\ Ulster University \\ Shore Rd., N'abbey \\ Co.Antrim, BT370QB \\ leopold-j@ulster.ac.uk
}

\author{
Philip A. Catherwood \\ Ulster University \\ Shore Rd., N'abbey \\ Co.Antrim, BT370QB \\ p.catherwood@ulster.ac.uk
}

\author{
James Uhomoibhi \\ Ulster University \\ Shore Rd., N'abbey \\ Co.Antrim, BT370QB \\ j.uhomoibhi@ulster.ac.uk
}

\begin{abstract}
This paper presents a novel machine learning prototype to actively assist hearing-impaired people during sleeping hours. The prototype can learn ambient sounds and intelligently decide if they are indicative of dangerous situations such as warning alarms, or if they are merely unimportant background sounds. The application is targeted towards the hearing-impaired who will have removed hearing aids or external components of cochlear implants before sleep. Any sounds of importance can then be translated into vibrations to awaken the sleeping hearing impaired user. The system is suggestive of future smart interactive machines to assist and protect those with either hearing loss or profound deafness.
\end{abstract}

Hearing, Intelligent, Machine learning, Prototype

\section{INTRODUCTION}

Around 11 million people in the United Kingdom are affected by hearing loss and is expected to rise to 15 million by 2035 [1]; $30 \%$ are children or adults of working age [2]. More than $5 \%$ of children aged 10 years or younger have hearing loss with $20 \%$ of people between the ages of 30-39 exhibiting significant hearing loss. Notably, almost $50 \%$ of people over 50 years present significant hearing problems [3].

Typically, at night time the hearing aid is removed from the ear canal and switched off, or in the case of those with cochlear implants the outer portion of the implant is removed. Therefore many hearing aid users cannot perceive such noises as raised voices, alarm clocks, smoke alarms, breaking glass, etc. The profoundly deaf often have suitably adapted environments with visual rather than aural indicators such as lights that flash when a door bell is pressed. For hearing aid users such adaptions are not normally required to allow them to lead active lives as the hearing devices assist them suitably during their waking hours; this ceases to be true when the device is removed at bedtime and exposes them to potential dangers to which they have no capacity to react.

This paper presents an early prototype device that uses machine learning to act as an intelligent environmental listening system for the deaf user while they sleep. The fully-functional prototype has been developed with the focus on detecting alarms initially (alarm clock and smoke alarm); this will be further developed to learn and compute a diverse array of sounds in the user's environment to awaken the hearing-impaired user on occasions that a hearing person would naturally awake (breaking glass, heavy rap on the door or window, running water, crying child, etc.). The device could sit against the user's pillow, be integrated into the mattress, or be worn on the wrist.

The device has specifically been designed with no wireless connection requirements (mobile phone, Wi-Fi link, etc.) as a correlation between wireless signals from mobile phones in proximity to a sleeping subject and increased brain stimulation has been established, specifically influences on the duration of each of the four natural stages of sleep cycle [3]. 


\section{PREVIOUS SOLUTIONS}

It is estimated that around 900,000 people in the UK have severe levels of hearing loss [1]. There are already diverse options on the market which cater for such difficulties. However, most of these solutions use bespoke doorbell systems, bespoke alarm systems, etc., most of which operate in isolation to each other.

Currently a number of systems and solutions attempt to meet the need of warning deaf people about audible warning alarms such as fire alarms [4], with some using complex multi-node systems that require permanent installation using pagerstyle vibrators [5] or a system of flashing lights and vibrating boxes [6]. In [7] a novel implant addresses the issue of night-time removal of the cochlear implant's external component and detects preprogrammed alarms creating a warning tone for the user; however such a solution requires surgical implantation and such technologies present a range of risks and issues [8, 9]. Additionally [10] offers an array of gadgets to address emergency situations as well as a range of morning alarm clocks. In every case the systems fail to incorporate advances in machine learning, having no learning capacity of their operational environment and often require professional installation.

The desire instead is to use unsupervised and autonomous auditory machine intelligence to learn and interpret everyday sounds; such sound classification has been previously explored for general usage [11, 12] as well as more specific machine learning to use audio to improve automatic video categorisation (Youtube) and indexing [13]. To date however such progress has not been translated into bespoke environmental machine learning for deaf users.

\section{PROTOTYPE DETAIL}

The device contains a miniature Adafruit microphone amplifier (1063) that operates over the bandwidth of natural healthy hearing and a microprocessor (32-pin surface mount ATMEGA328P)

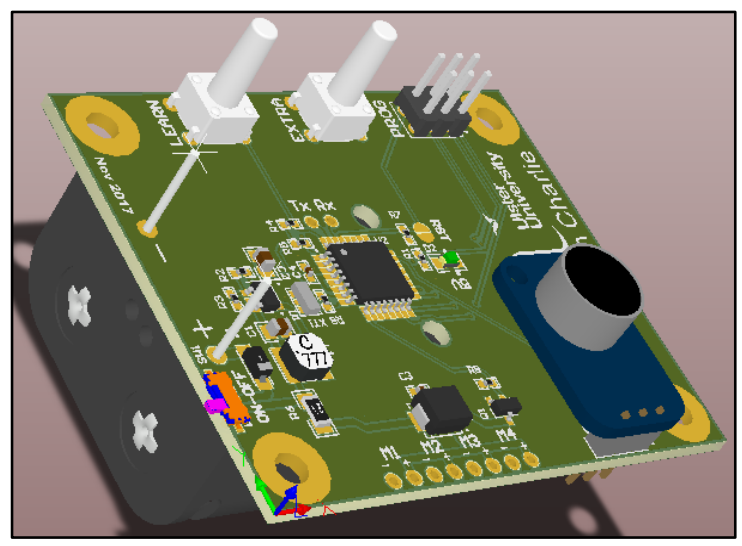

Figure 1: 3D computer model with embedded firmware. In operation the system will excite small vibrational motors to awaken the deaf user when a suitable sound is detected.

The main printed circuit board was developed using Altium Circuit Designer V17 for schematic capture and printed circuit board layout. Figure 1 depicts the 3D model of the finished board (which will be used to print a 3D bespoke casing), while Figure 2 presenting a photograph of the finished prototype.

The presented system has been initially tested using training alarm sounds (a range of stock mobile phone alarm sounds from an iPhone 7) then was tested against these same alarm sounds to determine the system's ability to determine whether the sound was an alarm or another sound.. In each test case the system correctly recognised the environmental audio input on the first occasion and excited the vibrational motors appropriately for correct stimulus. This work acts as a sanity check for the system's operability and as a datum for all further development activities.

Currently, learning is actuated by holding down the learn button; future iterations would intelligently learn sounds and decide if they are potentially useful as an alert trigger. A Fast Fourier Transform (FFT) of the sound is formed and written into the EEPROM memory of the microcontroller. The EEPROM ensures that trained sounds are still present after a battery change. Since the prototype utilises a low clock frequency $(16 \mathrm{MHz})$ with an 8bit processor a simplified FFT (Fast Hartley Transformation (FHT)) was used. This saves many repetitive calculations thus conserving resources on the microcontroller. Future prototype iterations will have a faster processor and more memory (Arm Cortex-M0+ processor). This provides enough computing power to calculate time-frequency spectra and compare against learned timefrequency bands, making it possible to detect more complicated acoustic signals. It is expected in

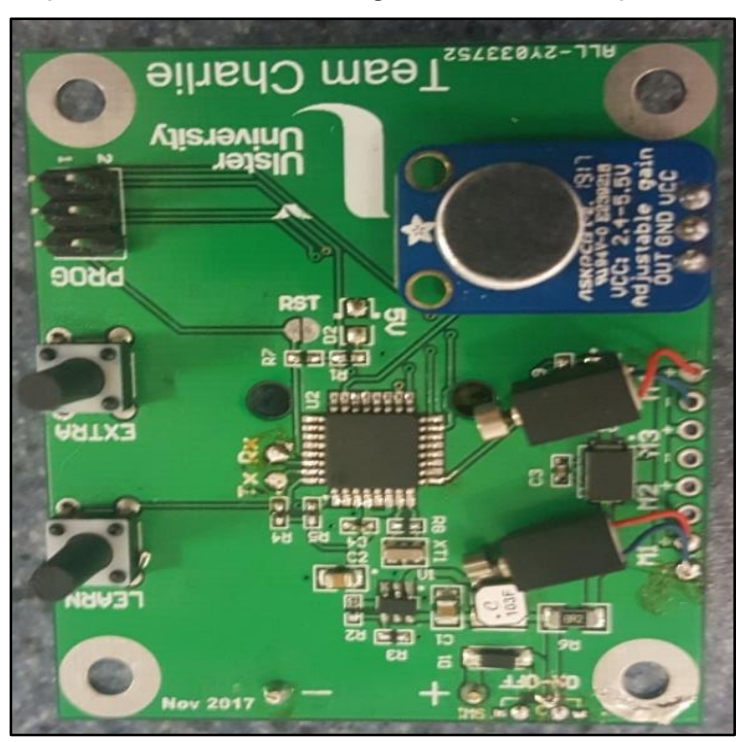

Figure 2: Prototyped print circuit board 
practical application that no two devices will react in the same way to the same stimuli as no two training environments are the same. Some standard sounds could be pre-installed (breaking glass) to avoid such a type of sound having to be learned in-situ.

\section{FURTHER TESTING AND DEVELOPMENT}

Further testing of known alarms will be undertaken to confirm the sensitivity (volume) and accuracy (number of false positives and negatives) of the system. Future iterations may use the hearing aid itself, the Amazon Alexa, or similar home-based system as the auditory learning device, which would acquire an array of known sounds to inform the smart nocturnal environment monitoring system. The vibrational feedback system could be programmed to vibrate in different modes or be linked with a reference display to indicate the exact nature of the significant sound.

With a trend towards wearables there may be opportunities to create miniature solutions, although wearables for such purposes may require significant trials to ensure their safety [14]; a device developed to protect hearing-impaired users cannot itself present a personal risk. Such wearable technology could furthermore be integrated with vital signs solutions to monitor blood oxygen levels [15] or electro-cardiograms (ECG) [16] to understand which sleep state the user is currently in [3] and select an optimal vibrational mode to most suitably awaken the user, avoiding either ineffectiveness or unnecessarily abrupt startling.

Such research may prove the potential for more advanced systems to aid those with total hearing loss where the system recognises individuals' voices, intonation, environmental sounds, etc. with perhaps the words or information being presented on a head-up display (HUD) or similar to allow the user to fully engage with their auditory surroundings. Such an intelligent system would require deep learning and significant processing power to realise real-time performance [17].

\section{REFERENCES}

[1] Speechbuddy. www.speechbuddy.com/blog/ hearing-loss/cochlear-implants-what-to-expectduring-surgery (10 Feb. 2018).

[2] hearinglink. www.hearinglink.org/your-hearing/ about-deafness-hearing-loss/facts-aboutdeafness-hearing-loss/ (10 Feb. 2018).

[3] Herer, G.R. (2013) American Speech Language Hearing Assoc. www.asha.org/Articles/ Intellectual-Disabilities-and-Hearing-Loss

$(10$ Feb. 2018).
[4] Bryan, V., Penney, G., Andrews, L., Russo M.R., (2014) Chapter 28: Empirical study using alternative early warning systems to address fire in the homes of deaf or hard-of-hearing individuals. Wang, V.C. (ed.), Handbook of R. on edu. and tech. changing soc. IGI Global, USA.

[5] Alert Group. www.alertergroup.com (14 Mar. 2018).

[6] Tynetec. www.tynetec.co.uk (14 Mar. 2018).

[7] Gordon, B., Leigh, R., Jurkiewicz, T., Tankongchumruskul, K. (2017) Wearable alarm system for a prosthetic hearing implant. US9630006B2, US Grant, 2017.

[8] Catherwood, P.A., Finlay, D.D., McLaughlin, J.A.D. (2015) Subcutaneous body area networks: A SWOT analysis. IEEE Intl. Symp. Technology and Society, Dublin Ireland, 11-12 Dec. 2015, pp. 1-8, IEEE.

[9] Catherwood, P.A., Finlay, D.D., McLaughlin, J.A.D. (2016) Intelligent Subcutaneous Body Area Networks: Anticipating Implantable Devices. IEEE Technology and Society Magazine, Vol. 35, pp. 73-80, IEEE.

[10] Action Hearing Loss. www.actiononhearingloss.org.uk (14 Mar. 2018)

[11] Salamon J., Bello, J.P. (2015) Unsupervised feature learning for urban sound classification. Intl. Conf. on Ac., Speech \& Sig. Proc., Brisbane, Australia, 19-24 April 2015, pp. 171-175, IEEE.

[12] Dieleman S., Schrauwen, B. (2014) End-to-end learning for music audio. Intl. Conf. on Acoustics, Speech and Signal Proc., Florence, Italy, 4-9 May 2014, pp. 6964-6968, IEEE.

[13] Hershey S. et al., (2017) CNN architectures for large-scale audio classification. Intl. Conf. on Acoustics, Speech and Signal Proc., New Orleans, LA, 5-9 Mar. 2017, pp. 131-135, IEEE.

[14] Harper, R. et al. (2010) Evaluation of a CE approved ambulatory patient monitoring device in a general medical ward. Ann. Intl. Conf. IEEE Eng. Med. \& Biol. Soc., Buenos Aires, Argentina, 31 Aug. - 4 Sep. 2010, pp. 94-97, IEEE.

[15] Schreiner, C., Catherwood, P., Anderson, J., McLaughlin, J. (2010) Blood oxygen level measurement with a chest-based pulse oximetry prototype system. Comp. in Cardiology, Belfast, UK, 26-29 Sept. 2010, pp. 537-540, IEEE.

[16] Catherwood, P.A., Donnelly, N., Anderson, J., McLaughlin, J. ECG motion artefact reduction improvements of a chest-based wireless patient monitoring system. Comp. in Cardiology, Belfast, UK, 26-29 Sept. 2010, pp. 557-560, IEEE.

[17] LeCun, Y., Bengio, Y., Hinton, G. (2015) Deep learning. Nature. vol. 521, pp. 436-444. 